\title{
Inflammatory role of high salt level in tumor microenvironment (Review)
}

\author{
SUNEETHA AMARA $^{1}$ and VENKATASWARUP TIRIVEEDHI ${ }^{2}$ \\ ${ }^{1}$ Department of Medicine, St. Thomas Health Mid-town Hospital; \\ ${ }^{2}$ Department of Biological Sciences, Tennessee State University, Nashville, TN, USA
}

Received February 4, 2017; Accepted March 22, 2017

DOI: $10.3892 /$ ijo.2017.3936

\begin{abstract}
Chronic inflammation is known to play a critical role in cancer development and progression. High salt is known to mediate several chronic inflammatory diseases including hypertension, myocardial infarction, neurological ischemic attack, autoimmune diseases and cancers. High salt level is shown to induce angiogenesis and immune-dysfunction, both of which play a direct role in cancer proliferation. Furthermore, salt has been suggested to enhance Warburg-like metabolic phenotype in cancer cells and at the same time also induce pro-tumor MФ2-macrophage phenotype. Recent studies have identified several molecular targets such as tonicity specific transcript factor NFAT5/TonEBP, sodium ion channel $\gamma \mathrm{ENaC}$, and vascular endothelial growth factor, VEGF, which are upregulated under high salt external environment. These molecular targets offer futuristic therapeutic application in precision medicine. In this review, we discuss the current understanding of the salt mediated metabolic and immune dysfunctions playing a potential role in cancerous changes.
\end{abstract}

\section{Contents}

1. Introduction

2. Impact of salt on cancer metabolism

3. Impact of salt on macrophages

4. Impact of salt on $\mathrm{CD} 4^{+} \mathrm{T}$ cells

5. Future perspectives

\section{Introduction}

Cancer is known to have multi-factorial etiology. Malignant transformation of terminally differentiated cells is characterized

Correspondence to: Dr Venkataswarup Tiriveedhi, Department of Biological Sciences, Tennessee State University, 3500 John A. Merritt Blvd., Nashville, TN 37209, USA

E-mail: vtirivee@tnstate.edu

Key words: inflammation, cytokines, macrophages, T-lymphocytes, Warburg effect by several key hallmarks that directly or indirectly lead to uncontrolled cell proliferation, reduced cell death, enhanced angiogenesis and metastatic potential $(1,2)$. Along with these more recently chronic inflammation is a well-recognized hallmark of cancer which is interestingly both a cause and effect of malignant transformation (3). The genetic events causing malignant transformation also initiate the expression of inflammation-related machinery that leads to development of a tumor inflammatory mileu (4). Oncogenes such as $R A S, M Y C$, and tyrosine kinases are known to induce proinflammatory cytokines and angiogenic signals (5). Conversely, chronic inflammation caused by carcinogens and infections is also known to induce the malignant genetic changes.

Epidemiologic studies increasingly support the notion that a strong positive correlation exits between chronic inflammatory diseases and risk of cancer development. Several critical molecular targets such as inducible nitric oxide synthase (iNOS), nuclear factor $\kappa \mathrm{B}(\mathrm{NF}-\kappa \mathrm{B})$ and hypoxiainducible factor- $1 \alpha$ (HIF-1 $\alpha$ ), have been identified to mediate the secretion of inflammatory cytokines (Fig. 1) that either directly or indirectly induce carcinogenesis (6). Although several inflammatory causes for cancers have been suggested environmental and dietary link is well established (7). High salt diet is well known to induce a pro-inflammatory cascade leading to chronic inflammatory diseases such as hypertension, myocardial infarction, neurological transient ischemic attack and cancer $(8,9)$. While the exact magnitude of salt-intake needed to induce inflammatory diseases is yet to be defined, however a cellular and immune dysregulation mediated by salt and potentially leading to cancer is well established and will be discussed in this review.

\section{Impact of salt on cancer metabolism}

Role of inflammatory sodium ion channels. An intratumor high salt concentration was demonstrated in early 1980s, when researchers demonstrated that the sodium content of breast tumors is significantly higher compared to a normal lactating breast epithelium (10). In these original studies it is unclear if the tumor activity is directly correlated with high intratumor salt concentration or if the high sodium concentration is extracellular or intracellular. Regardless, salt is known to induce inflammation. One of the well realized inflammatory markers, inducible nitric oxide synthases (iNOS) is shown to be upregu- 


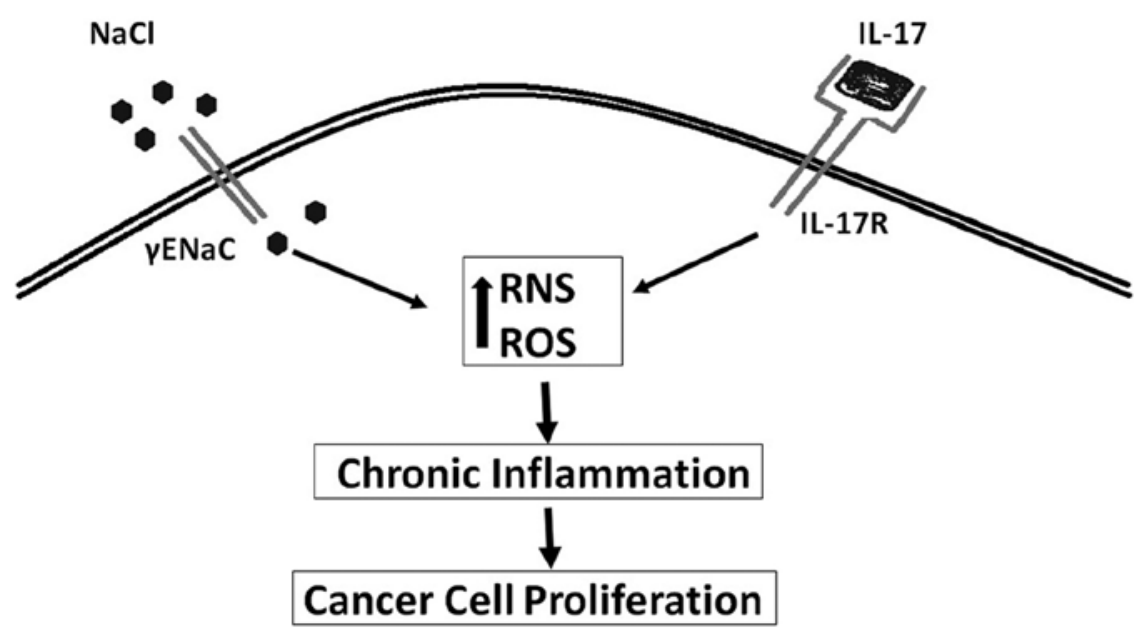

Figure 1. Synergistic pro-inflammatory effect of high salt and IL-17.

lated in several cancers including breast cancers (11). Our cellular studies on breast cancer cell lines MDA-MB-231 and MCF7 have shown that high sodium chloride concentration (0.05 $\mathrm{M}$ above basal level) was able to induce the production of reactive nitrogen and oxygen species (RNS/ROS) (12). Importantly an equimolar addition of mannitol to the media did not induce similar effect, thus suggesting sodium play a direct pro-inflammatory effect on breast cancer cell lines. Furthermore, earlier studies in 1980s in brain cancers have shown that an intracellular influx of sodium ions induced cancerous cell proliferation $(13,14)$. An increasing number of studies have shown that epithelial sodium channel (ENaC) which controls the intracellular entry of sodium ions is correlated with cancerous cell changes including, uncontrolled cell proliferation, anti-apoptosis and cell migration. Studies by Bondarava et al have demonstrated that $\mathrm{ENaC}$ played a critical role in the sodium induced cell proliferation of HepG2 cells (15). In line with this evidence, recent studies from our laboratory (Fig. 1) have demonstrated increased sodium concentration specifically induced upregulation of $\gamma \mathrm{ENaC}$ in the breast cancer cell lines and enhanced cellular proliferation (12). It is of interest to note that, the $\mathrm{ENaC}$ expression levels in breast cancer cell lines were upregulated by the mineralo-corticoid hormone aldosterone, known to control ionic equilibrium and not glucocorticoid steroid hormones (16). All these data suggest a direct functional role of $\mathrm{ENaC}$ in high-sodium induced cancerous development.

Role of salt in Warburg-metabolism. In a terminally differentiated cell, under normoxic conditions, glucose metabolism by mitochondrial oxidation yields 36-38 ATP. However, a cancerous cell under similar normoxic conditions, yields 2 ATP with subsequent metabolism of glucose to lactate. This aberrant behavior of cancerous cells is called aerobic glycolysis or Warburg effect named after the scientist Otto Warburg who originally discovered this phenomena in 1923 (17). This glycolytic switch in cancer cells has been used to explain their extraordinary ability to tolerate hypoxia and inflammatorystress. Furthermore, this glycolytic switch converts a catabolic glucose metabolism producing carbon dioxide to anabolic glucose metabolism resulting in higher lipid biosynthesis needed for cancerous cell proliferation (18). Osmotic stress induced by hyertonic saline has been suggested to induce Warburg-like effect by enhancing glucose transport and lactic acidosis in cancer cells (19). In line with these findings, our recent studies have demonstrated that high salt in the external environment induced Warburg-like effect of increased glucose consumption and lactic acid production in breast cancer cells (Fig. 2), which was abrogated by anti-inflammatory compound oleanolic acid (20). Taken together, these data suggest that high sodium plays a direct role in inducing cancerous metabolic phenotype.

Role of high salt in angiogenesis. Increased formation of new blood vessels to the newly formed tumor to support its growth and metabolism is considered as one of the important hallmarks of cancer development. Vascular endothelial growth factor (VEGF) is known to induce angiogenesis to tumors through activation of cancer-specific AKT/PI3k signaling mechanism (21). Recently strategies targeted against VEGF have been utilized in anticancer therapies (22). Our recent studies have demonstrated that high salt directly induces the expression of VEGF in breast cancer cells through the transcription factor (Fig. 3), nuclear factors of activated T cells or NFAT5 signaling (23). The NFAT5 also known as tonicity-responsive enhancer binding protein (TonEBP) is a well-established osmotic response element binding protein known to regulate intracellular osmotic tonicity (24). The NFAT5 is shown to exert a critical role in immune functioning and lymphocyte differentiation (25). Gene expression studies by Remo et al (26) on breast cancer patients $(n=197)$ have suggested a strong positive correlation between the expression of NFAT5 and inflammatory breast cancer. These accumulating data clearly suggest that high salt mediates a direct pro-angiogenic response in the tumor mileu.

\section{Impact of salt on macrophages}

The innate myeloid lineage macrophages play a critical role of antigen presentation and immune homeostasis. These terminally differentiated cells uniquely demonstrate a high degree of plasticity in response to changes in microenvironment. 
High Salt

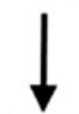

$\uparrow$ Aerobic glycolysis

$\downarrow$ Mitochondrial oxidation

$\downarrow$ Apoptosis

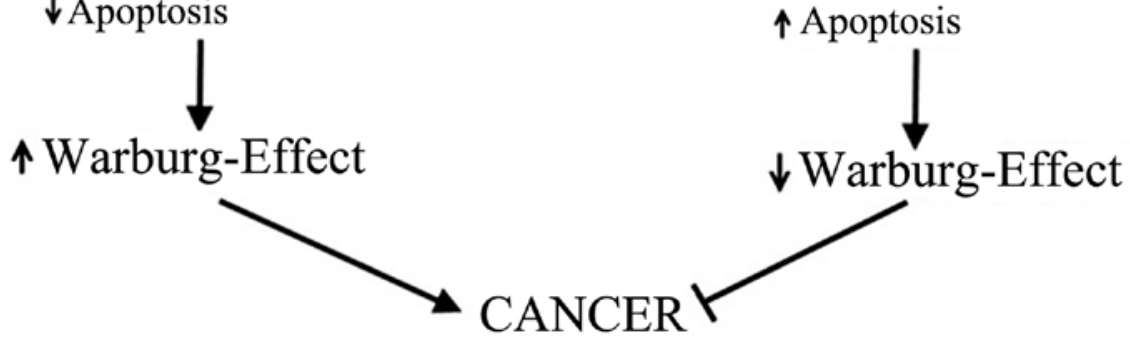

Figure 2. Enhanced Warburg-like phenomenon by high salt on cancer cells.

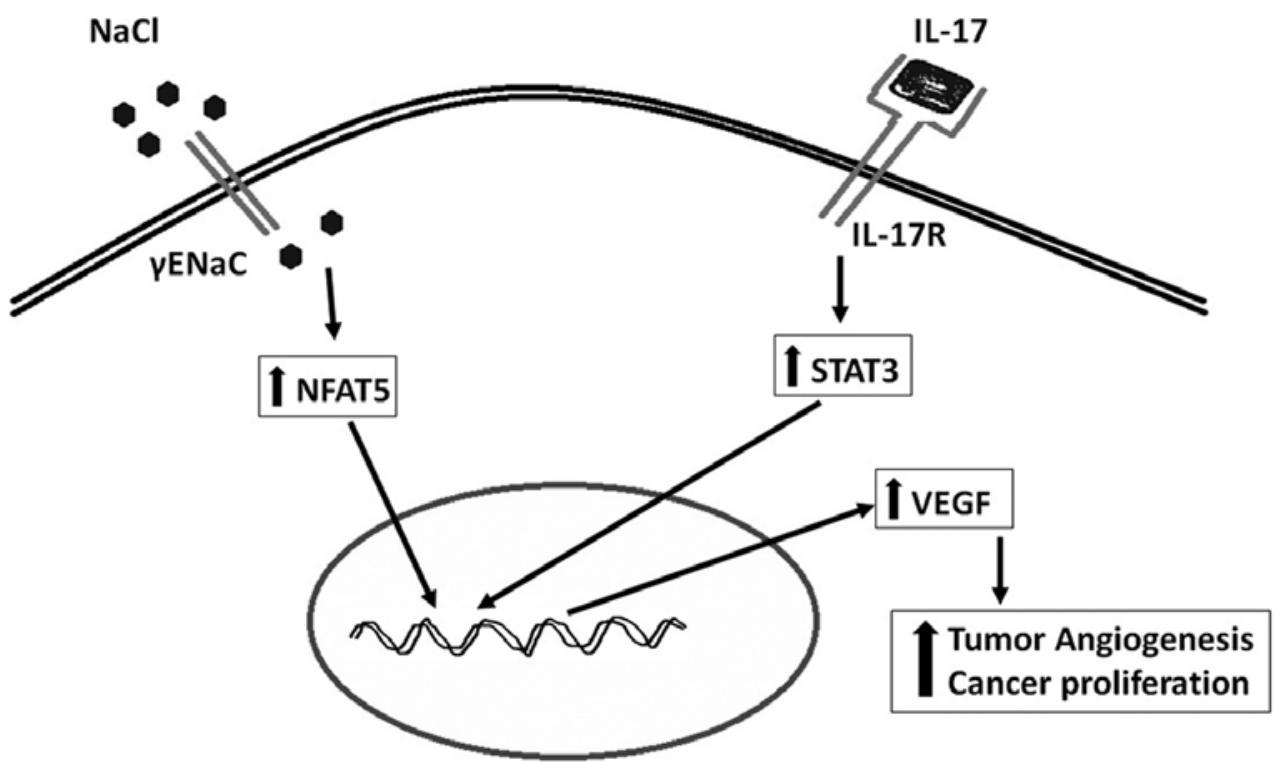

Figure 3. Potential synergistic effect of NFAT5 and STAT3 on cancer angiogenesis.

Two well recognized phenotypes of tissue-resident macrophages are classically activated pro-inflammatory type 1 macrophages (M1) and the alternatively-activated immunosuppressive type 2 macrophages (M2) (27). Various factors have been implicated in the phenotype switch of macrophages. The anti-inflammatory M2 phenotype is well established to be a resident phenotype in tumor TAMs (tumor associated macrophages) are shown to be negatively correlated with cancer prognosis. One of the signature cytokines released from TAMs is interleukin-10.

Studies in our laboratory have demonstrated that treatment with high salt in the culture media induced specific differentiation of peripheral blood mononuclear cells to M2 phenotype $\left(\mathrm{CD} 11 \mathrm{~b}^{+} \mathrm{CD} 14^{\text {low }} \mathrm{CD} 16^{+}\right)$. As tumors are known to have high salt microenvironment (28), our data strongly suggest that high salt induces anti-inflammatory M2 phenotype which are predominantly accumulated in tumor microenvironment (29). The cytokine array expressed in the tumor microenvironment is known to plays a critical role in the activation and differentiation of newly migrated mononuclear phagocytes (30). While, the M1 phenotype is induced by the cytokines interferon $\gamma(\mathrm{IFN} \gamma)$ and tumor necrosis factor $\alpha$ (TNF $\alpha)$ (31), the alternatively activated immune-suppressive TAM phenotype is induced by transforming growth factor $\beta$ (TGF $\beta$ ) in the cancer microenvironment (32). An important dissimilarity between MФ1 and M2 phenotypes is their cytokine pattern. The M1 phenotype secrets inflammatory cytokines (IL6, IL-1 $\beta$ and TNF $\alpha$ ) that are antitumor, while, M2 phenotype secretes pro-tumor IL-10, and VEGF (9). However, it seems the differentiation of tissue resident macrophages is also site-specific. Studies by Jantsch et al have demonstrated that, high salt activation macrophage induced an M1 phenotype switch offering protection against protozoan Leishmania skin infection (33). These data suggest that the high salt mediated activation of macrophage is tissue-specific and dependent on resident microenvironment. 


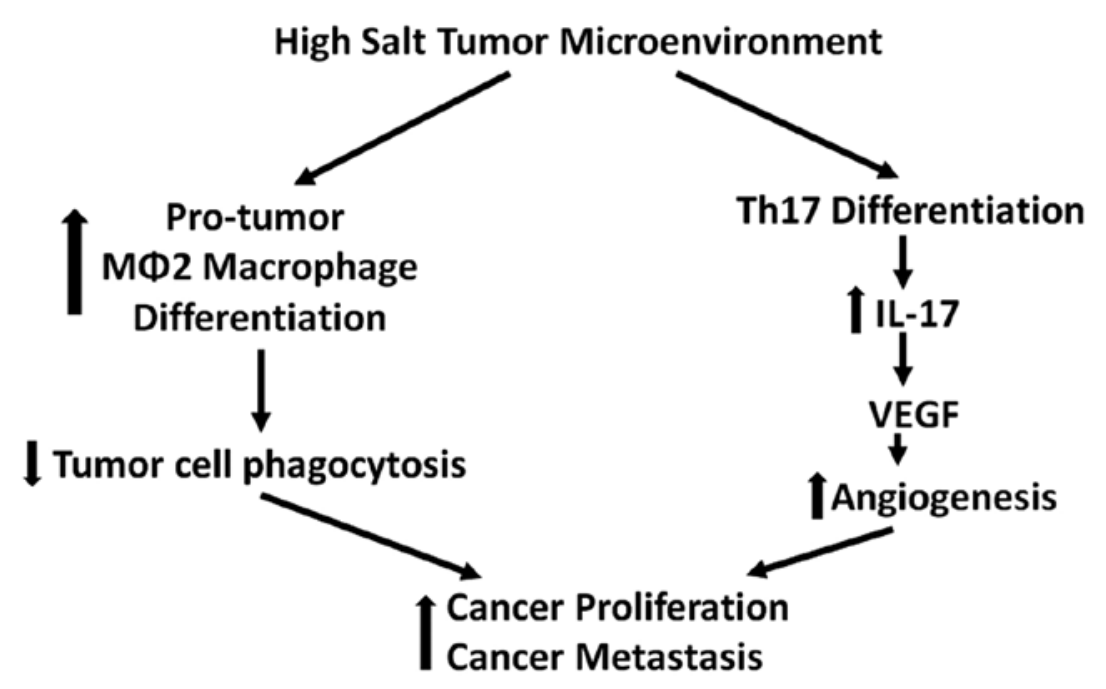

Figure 4. Pro-tumor effects of high salt mediated immune-modulation in tumor microenvironment.

\section{Impact of salt on $\mathrm{CD4}^{+} \mathrm{T}$ cells}

Local tumor inflammatory mileu is known to recruit several immune cells including $\mathrm{CD} 4^{+} \mathrm{T}$ helper (Th) lymphocytes. A subset of Th-cells which secrets a pro-inflammatory cytokine IL-17 is well established and is called Th17 phenotype (34). Several pre-clinical human cancer studies have demonstrated increased frequency of Th17 cells in the tumor infiltrating lymphocytes in several solid organ tumors $(35,36)$. The secretion of chemokines, MCP-1 and RANTES, by tumor resident fibroblasts have been suggested to play a key role in the recruitment of Th17 cells into the tumor (37). Along with these chemokines several other factors released by tumor cells such as Aryl hydrocarbon receptor (AhR) ligands, adenosine, and metabolites from hypoxia have been suggested to induce Th17 differentiation of naïve $\mathrm{CD}^{+}{ }^{+} \mathrm{Th}$ cells in the tumor microenvironment $(38,39)$. Furthermore, the newly recruited Th17 cells have been suggested to further induce tumor growth and metastasis (Fig. 4). Specifically, IL-17 has been demonstrated to induce pro-angiogenic VEGF in the tumor mileu leading to enhanced tumorigenicity and metastasis (40).

While, the precise mechanisms causing the Th17 differentiation in the tumor microenvironment are unclear, one possible hypothesis would be the possibility of high salt in the tumor microenvironment might influence differentiation of naïve $\mathrm{CD} 4^{+}$ T cells to Th17 phenotype. A very important indirect evidence to this effect, has been provided by $\mathrm{Wu}$ et al, where under in vitro conditions the researchers demonstrated that high salt in the culture media induced Th17 differentiation of naïve $\mathrm{CD} 4^{+}$ $T$ cells (41). In this study the authors have identified a salt-sensing transcription factor, serum-glucocorticoid kinase (SGK1), as the principal molecule it induces salt-specific differentiation to Th17 phenotype. The SGK1 knockdown in these experiments failed to induce salt-mediated Th17 differentiation.

Immunologically regulatory $\mathrm{T}$ cells (Tregs) have been shown to exert an antagonistic effect on Th17 differentiation and its molecular downstream effector function. The Treg cells are known to exert anti-inflammatory effects through secretion of immune-suppressive IL-10 cytokine. To further consolidate the notion that salt induces Th17 differentiation, studies by
Hernandez et al, demonstrated that high salt exerts inhibitory effect on the suppressive activity of Treg (42). In these studies, researchers demonstrated that the exposure of Treg to high salt inhibited the suppressive function along with increase in the pro-inflammatory factors such as IFNG, TBX21 and CXCR3. Furthermore, under these conditions there was an increase in the inflammatory IL-17 transcript confirming the studies by Wu et al (41) mentioned above. Furthermore, utilizing a humanized mouse model of graft versus host disease (GvHD) and a murine model of experimental colitis, Hernandez et al have shown that high salt induced an inflammatory $\mathrm{CD} 4^{+} \mathrm{T}$ cell immunophenotype and worsening of the disease (42). However, a direct study on the role of salt on the tumor will be needed to confirm the effect of salt on tumors.

\section{Future perspectives}

High salt diet is known to be the key culprit in several chronic inflammatory diseases including cardiovascular disease, cancer and autoimmune diseases. A typical adult Western diet contains $10-12 \mathrm{~g}$ sodium per day which is 2-3 times above the WHO recommended $4.8 \mathrm{~g}$ of sodium per day (43). Several epidemiological studies have conclusively supported a positive correlation between salt and cancer. A wide-spread awareness on saltrestricted diet might be able to reduce the community disease load. Recent pre-clinical and clinical studies have identified several molecular targets which mediate salt-induced damage. These molecular targets could offer novel futuristic intervention treatment strategies for personalized precision medicine.

\section{References}

1. Hanahan D and Weinberg RA: The hallmarks of cancer. Cell 100: 57-70, 2000 .

2. Balkwill F and Mantovani A: Inflammation and cancer: Back to Virchow? Lancet 357: 539-545, 2001.

3. Mantovani A, Allavena P, Sica A and Balkwill F: Cancer-related inflammation. Nature 454: 436-444, 2008.

4. Borrello MG, Alberti L, Fischer A, Degl'innocenti D, Ferrario C, Gariboldi M, Marchesi F, Allavena P, Greco A, Collini P, et al: Induction of a proinflammatory program in normal human thyrocytes by the RET/PTC1 oncogene. Proc Natl Acad Sci USA 102: 14825-14830, 2005. 
5. Ischenko I, Zhi J, Moll UM, Nemajerova A and Petrenko O Direct reprogramming by oncogenic Ras and Myc. Proc Nat Acad Sci USA 110: 3937-3942, 2013.

6. Lu H, Ouyang $\mathrm{W}$ and Huang C: Inflammation, a key event in cancer development. Mol Cancer Res 4: 221-233, 2006.

7. Wang XQ, Terry PD and Yan H: Review of salt consumption and stomach cancer risk: Epidemiological and biological evidence. World J Gastroenterol 15: 2204-2213, 2009.

8. Aburto NJ, Ziolkovska A, Hooper L, Elliott P, Cappuccio FP and Meerpohl JJ: Effect of lower sodium intake on health: Systematic review and meta-analyses. BMJ 346 (apr03 3): f1326, 2013.

9. Ge S, Feng X, Shen L, Wei Z, Zhu Q and Sun J: Association between habitual dietary salt intake and risk of gastric cancer: A systematic review of observational studies. Gastroenterol Res Pract 2012: 808120, 2012

10. Sparks RL, Pool TB, Smith NK and Cameron IL: Effects of amiloride on tumor growth and intracellular element content of tumor cells in vivo. Cancer Res 43: 73-77, 1983.

11. Lechner M, Lirk P and Rieder J: Inducible nitric oxide synthase (iNOS) in tumor biology: The two sides of the same coin. Semin Cancer Biol 15: 277-289, 2005.

12. Amara S, Ivy MT, Myles EL and Tiriveedhi V: Sodium channel $\gamma \mathrm{ENaC}$ mediates IL-17 synergized high salt induced inflammatory stress in breast cancer cells. Cell Immunol 302: $1-10,2016$

13. O'Donnell ME, Cragoe E Jr and Villereal ML: Inhibition of $\mathrm{Na}^{+}$ influx and DNA synthesis in human fibroblasts and neuroblastoma-glioma hybrid cells by amiloride analogs. J Pharmacol Exp Ther 226: 368-372, 1983

14. O'Donnell ME and Villereal ML: Membrane potential and sodium flux in neuroblastoma X glioma hybrid cells: Effects of amiloride and serum. J Cell Physiol 113: 405-412, 1982.

15. Bondarava M, Li T, Endl $\mathrm{E}$ and Wehner F: alpha-ENaC is a functional element of the hypertonicity-induced cation channel in HepG2 cells and it mediates proliferation. Pflugers Arch 458: 675-687, 2009.

16. Masilamani S, Kim GH, Mitchell C, Wade JB and Knepper MA Aldosterone-mediated regulation of $\mathrm{ENaC}$ alpha, beta, and gamma subunit proteins in rat kidney. J Clin Invest 104: R19-R23, 1999.

17. Maldonado EN and Lemasters JJ: ATP/ADP ratio, the missed connection between mitochondria and the Warburg effect. Mitochondrion 19 Pt A: 78-84, 2014.

18. Palsson-McDermott EM and O'Neill LA: The Warburg effect then and now: From cancer to inflammatory diseases. BioEssays 35: 965-973, 2013

19. Epstein T, Xu L, Gillies RJ and Gatenby RA: Separation of metabolic supply and demand: Aerobic glycolysis as a norma physiological response to fluctuating energetic demands in the membrane. Cancer Metab 2: 7, 2014

20. Amara S, Zheng M and Tiriveedhi V: Oleanolic acid inhibits high salt-induced exaggeration of Warburg-like metabolism in breast cancer cells. Cell Biochem Biophys 74: 427-434, 2016

21. Pidgeon GP, Barr MP, Harmey JH, Foley DA and BouchierHayes DJ: Vascular endothelial growth factor (VEGF) upregulates BCL-2 and inhibits apoptosis in human and murine mammary adenocarcinoma cells. Br J Cancer 85: 273-278, 2001.

22. Harmey JH and Bouchier-Hayes D: Vascular endothelial growth factor (VEGF), a survival factor for tumour cells: Implications for anti-angiogenic therapy. BioEssays 24: 280-283, 2002.

23. Amara S, Alotaibi D and Tiriveedhi V: NFAT5/STAT3 interaction mediates synergism of high salt with IL-17 towards induction of VEGF-A expression in breast cancer cells. Oncol Lett 12 933-943, 2016

24. Neuhofer W: Role of NFAT5 in inflammatory disorders associated with osmotic stress. Curr Genomics 11: 584-590, 2010.
25. Berga-Bolaños R, Drews-Elger K, Aramburu J and LópezRodríguez C: NFAT5 regulates T lymphocyte homeostasis and CD24-dependent $\mathrm{T}$ cell expansion under pathologic hypernatremia. J Immunol 185: 6624-6635, 2010.

26. Remo A, Simeone I, Pancione M, Parcesepe P, Finetti P, Cerulo L, Bensmail H, Birnbaum D, Van Laere SJ, Colantuoni V, et al: Systems biology analysis reveals NFAT5 as a novel biomarker and master regulator of inflammatory breast cancer. J Transl Med 13: 138, 2015.

27. Quatromoni JG and Eruslanov E: Tumor-associated macrophages: Function, phenotype, and link to prognosis in human lung cancer. Am J Transl Res 4: 376-389, 2012.

28. Roger S, Besson P and Le Guennec JY: Involvement of a novel fast inward sodium current in the invasion capacity of a breast cancer cell line. Biochim Biophys Acta 1616: 107-111, 2003.

29. Amara S, Whalen M and Tiriveedhi V: High salt induces antiinflammatory $\mathrm{M} \Phi 2$-like phenotype in peripheral macrophages. Biochem Biophys Rep 7: 1-9, 2016.

30. Solinas G, Germano G, Mantovani A and Allavena P: Tumorassociated macrophages (TAM) as major players of the cancer-related inflammation. J Leukoc Biol 86: 1065-1073, 2009.

31. Lewis CE and Pollard JW: Distinct role of macrophages in different tumor microenvironments. Cancer Res 66: 605-612, 2006.

32. Colotta F, Allavena P, Sica A, Garlanda C and Mantovani A Cancer-related inflammation, the seventh hallmark of cancer: Links to genetic instability. Carcinogenesis 30: 1073-1081, 2009.

33. Jantsch J, Schatz V, Friedrich D, Schröder A, Kopp C, Siegert I, Maronna A, Wendelborn D, Linz P, Binger KJ, et al: Cutaneous $\mathrm{Na}^{+}$storage strengthens the antimicrobial barrier function of the skin and boosts macrophage-driven host defense. Cell Metab 21: 493-501, 2015.

34. Guéry L and Hugues S: Th17 cell plasticity and functions in cancer immunity. BioMed Res Int 2015: 314620, 2015.

35. Wang L, Yi T, Kortylewski M, Pardoll DM, Zeng D and Yu H: IL-17 can promote tumor growth through an IL-6-Stat3 signaling pathway. J Exp Med 206: 1457-1464, 2009.

36. Bailey SR, Nelson MH, Himes RA, Li Z, Mehrotra S and Paulos CM: Th17 cells in cancer: The ultimate identity crisis. Front Immunol 5: 276, 2014.

37. Su X, Ye J, Hsueh EC, Zhang Y, Hoft DF and Peng G: Tumor microenvironments direct the recruitment and expansion of human Th17 cells. J Immunol 184: 1630-1641, 2010

38. Kimura A, Naka T, Nohara K, Fujii-Kuriyama Y and Kishimoto T: Aryl hydrocarbon receptor regulates Stat1 activation and participates in the development of Th17 cells. Proc Natl Acad Sci USA 105: 9721-9726, 2008

39. Wilson JM, Kurtz CC, Black SG, Ross WG, Alam MS, Linden J and Ernst PB: The A2B adenosine receptor promotes Th17 differentiation via stimulation of dendritic cell IL-6. J Immunol 186: 6746-6752, 2011.

40. Wu X, Yang T, Liu X, Guo JN, Xie T, Ding Y, Lin M and Yang H: IL-17 promotes tumor angiogenesis through Stat3 pathway mediated upregulation of VEGF in gastric cancer. Tumour Biol 37: 5493-5501, 2016

41. Wu C, Yosef N, Thalhamer T, Zhu C, Xiao S, Kishi Y, Regev A and Kuchroo VK: Induction of pathogenic TH17 cells by inducible salt-sensing kinase SGK1. Nature 496: 513-517, 2013.

42. Hernandez AL, Kitz A, Wu C, Lowther DE, Rodriguez DM, Vudattu N, Deng S, Herold KC, Kuchroo VK, Kleinewietfeld M, et al: Sodium chloride inhibits the suppressive function of $\mathrm{FOXP}^{+}$regulatory T cells. J Clin Invest 125: 4212-4222, 2015.

43. Cordain L, Eaton SB, Sebastian A, Mann N, Lindeberg S, Watkins BA, O'Keefe JH and Brand-Miller J: Origins and evolution of the Western diet: Health implications for the 21st century. Am J Clin Nutr 81: 341-354, 2005. 